\title{
Effect of penile rehabilitation with low intensity extracorporeal shock wave therapy on erectile function recovery following robot- assisted laparoscopic prostatectomy
}

\author{
Shogo Inoue, Tetsutaro Hayashi, Jun Teishima, Akio Matsubara \\ Department of Urology, Graduate School of Biomedical and Health Sciences, Hiroshima University, Hiroshima, Japan \\ Contributions: (I) Conception and design: S Inoue; (II) Administrative support: J Teishima, A Matsubara; (III) Provision of study materials or patients: \\ S Inoue, J Teishima; (IV) Collection and assembly of data: S Inoue, T Hayashi; (V) Data analysis and interpretation: S Inoue, J Teishima; (VI) \\ Manuscript writing: All authors; (VII) Final approval of manuscript: All authors. \\ Correspondence to: Shogo Inoue. Department of Urology, Graduate School of Biomedical and Health Sciences, Hiroshima University, Hiroshima, \\ Japan. Email: inosyogo@hiroshima-u.ac.jp
}

Backgrounds: Considering the natural course of cavernous nerve recovery after robot-assisted laparoscopic prostatectomy (RALP), early intervention of low intensity extracorporeal shock wave therapy (LIESWT) would be more effective for enhancing overall recovery of sexual function (SF). Our objective of this study is to analyze longitudinally the alterations of SF in patients after RALP, with a focus on the effect of early and delayed intervention with LIESWT.

Methods: A total of 5 and 11 patients underwent early and delayed intervention with LIESWT, respectively. SF was assessed with the Expanded Prostate Cancer Index Composite (EPIC). The same surgeon performed RALP on 178 patients, and these patients were assigned to the non-LIESWT group to establish a control group. The SF score of EPIC was investigated longitudinally before RALP and 3, 6, 9, and 12 months after RALP.

Results: Our results show that penile rehabilitation with LIESWT immediately before urethral catheter removal improved SF scores. In the baseline, the SF score was significantly higher in the early LIESWT group ( $\mathrm{P}=0.0001)$. The SF score was significantly lower at postoperative 6 months (early 19.2, delayed 17.9, and non-LIESWT 8.1; $\mathrm{P}=0.0171), 9$ months (20.9, 25.8, and 10.2; $\mathrm{P}=0.0188)$, and 12 months (28.0, 21.3, and 9.5; $\mathrm{P}=0.0051$ ) in the non-LIESWT group. We regret that there was no significant difference in the recovery of SF between the early and delayed protocol with LIESWT at all points. In keeping with our results, LIESWT demonstrated the potential to be efficacious in treatment options for severe postradical prostatectomy $(\mathrm{RP})$ erectile dysfunction $(\mathrm{ED})$ as it may indirectly support its promotion of nerve regeneration in severe $\mathrm{ED}$ due to $\mathrm{RP}$.

Conclusions: This is the first study in which LIESWT has been shown to deliver a clinical benefit on its early or delayed intervention to patients after RALP to penile rehabilitation in terms of restoring SF. Our preliminary results suggest that LIESWT could be used as a treatment option in penile rehabilitation.

Keywords: Low intensity extracorporeal shock wave therapy (LIESWT); sexual function; radical prostatectomy; penile rehabilitation

Submitted Dec 19, 2019. Accepted for publication May 25, 2020.

doi: 10.21037/tau-19-888

View this article at: http://dx.doi.org/10.21037/tau-19-888 


\section{Introduction}

Erectile dysfunction (ED) is one of the most common disorders in middle-aged men, and this disease considerably influences quality of life (QOL) (1). Currently, the main non-surgical treatment for ED is the oral administration of phosphodiesterase type 5 inhibitors (PDE5I). These drugs were very effective and common treatments (2), but were determined to be only on-demand palliative management in treatment decisions for patients with ED. The ideal endpoint should be the rehabilitation of patients with ED to potentially recover from corpus cavernosum pathological changes, enabling spontaneous sexual function (SF) with few side effects (3). Low intensity extracorporeal shock wave therapy (LIESWT) has proven to be effective and safe for various types of medical disorders such as angiogenesis in chronic diabetic foot ulcers and myocardial ischemia $(4,5)$.

Growing public concern with prostate cancer $(\mathrm{PCa})$ coupled with the dissemination of prostate-specific antigen (PSA) checks has induced younger men to diagnose PCa early, enabling early treatment. Presently, patients with localized PCa have more potential to have long life expectancies after radical prostatectomy (RP). SF was drastically decreased at three months and slightly increased one year after RP (6), Younger men with normal preoperative $\mathrm{SF}$ who have received bilateral nerve sparing (NS)-RP (7-9) could potentially fully recover after four years. However, $20-80 \%$ of these patients may never recover to a normal level (10).

Considering the natural course of cavernous nerve recovery after robot-assisted laparoscopic prostatectomy (RALP), early intervention of LIESWT would be more effective for enhancing overall recovery of SF. This is because early intervention might supply blood flow to the corporeal bodies more effectively during temporary neuropraxia in the cavernous nerve, occurring immediately after RP. However, to the best of our knowledge, it has not been clearly proven in practical settings whether early rehabilitation of LIESWT immediately after RP improves erectile function recovery more efficiently than delayed intervention (11). We analyzed longitudinally alterations in $\mathrm{SF}$ and sexual bother (SB) of the Expanded Prostate Cancer Index Composite (EPIC) in patients after RALP, with a focus on the effect of early and delayed intervention with LIESWT. We present the following article in accordance with the STROBE reporting checklist (available at http:// dx.doi.org/10.21037/tau-19-888).

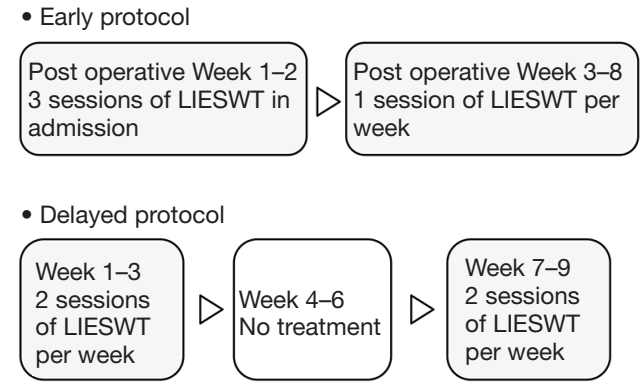

Figure 1 Early and delayed LIESWT treatment protocol.

\section{Methods}

The principles of the Helsinki Declaration (as revised in 2013) were followed during the study, and the confidentiality of the patients' data was guaranteed. The study was approved by the Institutional Review Board of Hiroshima University Hospital (NO. C-87) and informed consent was taken from all the patients. Five patients after RALP were enrolled into this study's protocol over a period between January and June 2017. We excluded patients (I) with an unstable psychiatric or medical condition, (II) with a past history of a neurological pathology, and (III) with a past history of PDE5I medication.

Our protocol was modified from that reported by Vardi et al. (12). LIESWT was performed by a probe attached to a compact electrohydraulic unit with a focused shock wave source (Omnispec ED1000; Medispec, Germantown, MD, USA) during each session. The penis was manually stretched, and shock waves were delivered to five sites: the distal, mid and proximal penile shaft, and both sides of the bilateral crura. The treatment time of each LIESWT session was approximately 20 minutes, and each session consisted of 300 shocks for each treatment site $(1,500$ per session) at an energy density of $0.09 \mathrm{~mJ} / \mathrm{mm}^{2}$ and a frequency of $120 / \mathrm{min}$. The volume of penile tissue exposed to the shock waves at each site was cylindrical. Local or systemic analgesia was not necessary during this procedure. This early intervention was performed three times a week for two weeks in admission and one time per week in our out-patient clinic for six weeks (Figure 1).

To establish a control group, the same surgeon performed RALP on 11 patients over a period between June 2012 and December 2014. These patients received LIESWT and this delayed protocol was same as that by Vardi et al. (Figure 1). The same surgeon performed RALP on 178 patients over a period between April 2012 and 
Table 1 Baseline patient characteristics and surgical outcome classified by LIESWT

\begin{tabular}{|c|c|c|c|c|}
\hline Characteristics & Early & Delayed & Non-LIESWT & $P$ value \\
\hline Age, yr & $62.2 \pm 2.68$ & $62.9+1.80$ & $66.6+0.45$ & 0.0453 \\
\hline $\mathrm{BMI}, \mathrm{kg} / \mathrm{m}^{2}$ & $24.5 \pm 1.25$ & $23.3 \pm 0.84$ & $23.4 \pm 0.21$ & 0.6768 \\
\hline $\mathrm{PSA}, \mathrm{ng} / \mathrm{mL}$ & $4.95 \pm 2.82$ & $6.39 \pm 1.90$ & $9.12 \pm 0.47$ & 0.1455 \\
\hline Prostate volume, $\mathrm{mL}$ & $25.2 \pm 5.76$ & $23.1 \pm 3.88$ & $29.2 \pm 0.97$ & 0.2657 \\
\hline SHIM & $17.8 \pm 3.52$ & $17.2 \pm 2.37$ & $11.4 \pm 0.65$ & 0.0178 \\
\hline Nerve sparing, \% & 20.0 & 63.6 & 43.8 & 0.2252 \\
\hline Surgical time, $\min$ & $213.6 \pm 19.1$ & $192.0 \pm 12.8$ & $201.7 \pm 3.2$ & 0.6209 \\
\hline
\end{tabular}

February 2015. These patients were assigned to the nonLIESWT group to establish another control group. SF and SB were assessed with the EPIC. A validated form of the self-reported health-related QOL questionnaire was administered to the patients before RALP, and mailed to all patients receiving RALP, with a stamped return envelope at 3, 6, 9, and 12 months after RALP. Demographic and historical data obtained included age, body mass index (BMI), PSA, serum Testosterone, prostate volume, Sexual Health Inventory for Men (SHIM) score, ratio of nerve sparing procedure, surgical time, and estimated blood loss.

We analyzed the survey data for each group by using the qui-square test, Manne-Whitney $\mathrm{U}$ test, and one-way analysis of variance (ANOVA). Each analysis was two-tailed; in all tests, $\mathrm{P}$ values $<0.05$ were decided to be statistically significant. All values were described as the mean, and the results were analyzed by the Mann-Whitney $\mathrm{U}$ test and one-way ANOVA. All survey data was analyzed using the JMP version 10 statistical software package (SAS Institute Inc., Cary, NC, USA).

\section{Results}

None of the patients complained of any pain or adverse events such as ecchymosis or hematuria due to or after the LIESWT in this study. Table 1 summarizes the details of the baseline patient characteristics and surgical outcomes between the three groups (early, delayed, and nonLIESWT). The mean duration from RALP to the start of penile rehabilitation was 6 months for patients of the delayed LIESWT group. In the non-LIESWT group, age was significantly higher $(\mathrm{P}=0.0453)$ and the preoperative SHIM score was significantly lower $(\mathrm{P}=0.0178)$.

Of the 194 enrolled subjects, 194, 166, 137, 120, and 115 patients completed the questionnaires at preoperative, $3,6,9$, and 12 postoperative months, respectively, which corresponds to response rates of $100 \%, 86 \%, 71 \%, 62 \%$, and $59 \%$, respectively. In the case with LIESWT, response rates were $100 \%, 94 \%, 88 \%, 69 \%$, and $63 \%$, respectively. These deficient data were appropriately treated in this evaluation. In the baseline, the SF score was $66.5,41.8$, and 31.8 in the early, delayed, and non-LIESWT group, respectively. The SF score was significantly higher at baseline in the early LIESWT group $(\mathrm{P}=0.0001)$ and significantly lower at postoperative 6 months (early 19.2, delayed 17.9, and non-LIESWT 8.1; $\mathrm{P}=0.0171), 9$ months (20.9, 25.8, and 10.2; $\mathrm{P}=0.0188)$, and 12 months $(28.0,21.3$, and $9.5 ; \mathrm{P}=0.0051$ ) in the non-LIESWT group. However, there was no significant difference in SF score between the early and delayed LIESWT groups at all points (Figure 2).

In the baseline, the SB score was $86.3,69.9$, and 87.9 in the early, delayed, and non-LIESWT group, respectively. The SB score was significantly lower at baseline in the delayed delayed LIESWT group $(\mathrm{P}=0.0070)$ and significantly higher at postoperative 3 months (early 33.8, delayed 41.9, and non-LIESWT 68.3; $\mathrm{P}=0.0045$ ) in the non-LIESWT group. However, the SB score in both the early and delayed LIESWT groups increased from postoperative 6 months, so there was no significant difference between the three groups at postoperative 6 months (early 46.3, delayed 54.2, and non-LIESWT 71.3; $\mathrm{P}=0.0886), 9$ months $(61.3,71.9$, and 68.9; $\mathrm{P}=0.8540)$, and 


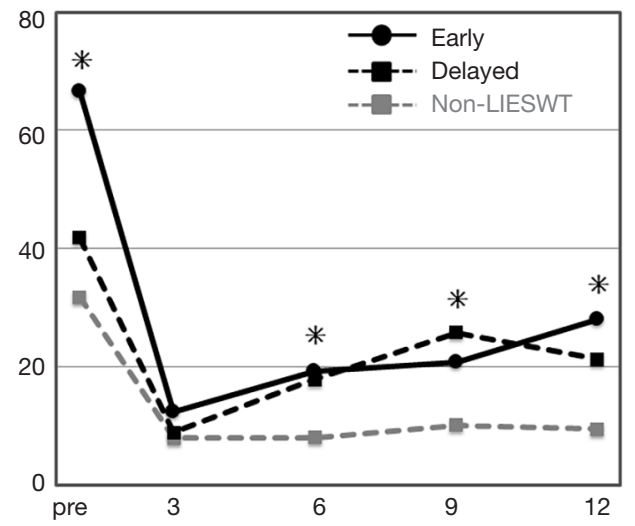

Figure 2 Longitudinal changes in SF scores before and after RALP in accordance with LIESWT.

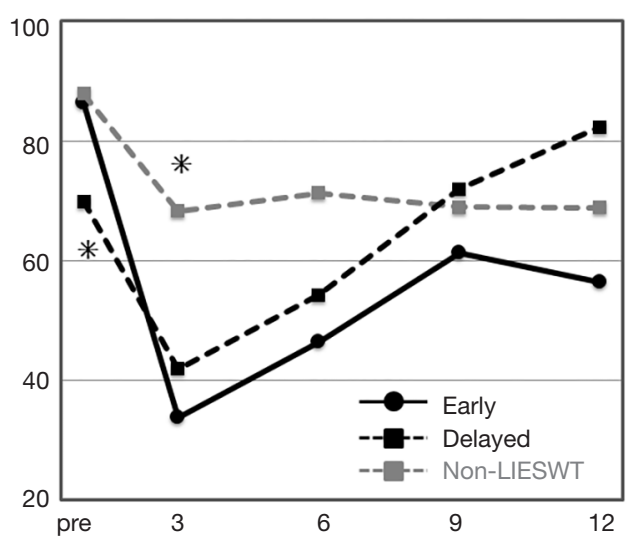

Figure 3 Longitudinal changes in SB scores before and after RALP in accordance with LIESWT.

12 months (56.3, 82.3, and 68.8; $\mathrm{P}=0.4298)$ (Figure 3).

\section{Discussion}

The first application of high intensity shock waves was reported in 1980. This novel treatment of extracorporeal shock wave lithotripsy was applied to the treatment of renal stones without any incisional or abdominal surgery (13). These applications of LIESWT were applied in various medical fields and so this treatment technology was recognized to be a revolution. A number of treatment devices use mechanical stress to generate acoustic waves to carry energy to targeted and focused deep-tissue areas, and are subsequently used to treat pancreatic stones, gall stones, and even nonunions and pseudoarthrosis (14-18).

In-vitro studies have shown that various growth factors were stimulated by LIESWT. The efficacy of LIESWT was reported for a patient with a cardiovascular disease when it was shown to promote angiogenesis by upregulating the expression of growth factors such as the vascular endothelial growth factor (4). When LIESWT is applied to a tissue, the relatively weak yet focused shock waves interact with targeted deep tissue causing microtrauma and mechanical stress, also known as shear stress (19). This phenomenon then triggers a series of events that cause the release of angiogenic factors, inducing angiogenesis of targeted vessels and enhancing blood flow. Extrapolation of these findings to ED has led to the assumption that if applied to the corpora cavernosa, LIESWT could ameliorate penile blood flow and endothelial function by stimulating neovascularization in the penile tissue (3).

Gruenwald et al. reported that LIESWT could be a potential treatment option for patients with severe ED who responded poorly to PDE5I treatment (20). After administration, the mean International Index of Erectile Function-Erectile Function domain (IIEFEF) scores increased and a significant improvement in penile hemodynamics was found. There were no severe complications reported during or after this clinical trial. From their study, it was suggested that such a unique modality of LIESWT could expand novel urological treatment options in the management of severe ED or poor responders to PDE5I.

We have faced a challenging and severe problem of postRP ED. Although NS surgical procedures have been used, it was reported that 12- and 24-month erection rates ranged from $54 \%$ to $90 \%$ and from $63 \%$ to $94 \%$, respectively (21). Current randomized-controlled trials have mainly covered vasculogenic ED. However, the exclusion of patients with post-RP ED in these studies raises a number of questions. We recognized that post-RP ED is mainly caused by injured neurovascular bundles (NVBs) and RP can temporarily or permanently damage the NVBs. Neuropraxia is the main cause of ED even after bilateral NS RP, as a result of intraoperative manipulations such as traction, compression, and coagulation (22). The injury of the cavernous nerve may causes temporary nervous degeneration, and subsequent denervation of the corpora cavernosa. Long-term penile hypoxia consequently causes penile structural remodeling with smooth muscle apoptosis, fibrosis, and sinusoidal obstruction (23).

Various treatment approaches have been reported in expectation of the efficacy of penile rehabilitation. However, these treatment strategies are still considered 
to be controversial from the point of view of restoring SF. The primary and original methods are (I) regular oral administration of PDE5I, (II) intracavernosal injection of Prostaglandin E1, and (III) the use of vacuum constriction devices (24). Many reviews of the latest studies concerned with penile rehabilitation came to the conclusion that although theoretical considerations recommend early intervention of penile rehabilitation to enable cavernous oxygenation, there is little clinical evidence to support the use of current protocols (25). However, no easily administered effective treatment of ED after RP has yet emerged (24). The potential of LIESWT, which enables both erection recovery and increased blood flow to the penile tissue, could enable useful intervention in penile rehabilitation due to RP.

LIESWT focuses on vasculogenic disorders as the primary research target in clinical settings, and research on post-RP ED is very rare. Current evidence that robustly supports the application of LIESWT for post-RP ED is therefore lacking (26). It was suggested that an ED patient after non-NS RP did not recover their SF using LIESWT postoperatively (27). The capacity of LIESWT to improve the outcome of SF after nerve injury has been observed in neurological diseases. It is considered that its neuroprotective and/or regenerative effects are related to modulated inflammation, local angiogenesis, continuous expression of neuropathic factors, and reduction of free radicals. However, the precise mechanisms of LIESWT are still unknown and under investigation.

The introduction of LIESWT may affect the recovery of erectile function in patients with post-RP ED (26). Early use of LIESWT as a part of penile rehabilitation should be aimed at accelerating neural recovery, improving cavernosal oxygenation, and preventing penile remodeling relying on the pathological findings of penile tissues after RP. On the other hand, late use of LIESWT, especially 2 or more months after RP, should be mainly aimed at reversing penile fibrotic remodeling (28). It was reported that treatments were introduced between 48 and $72 \mathrm{~h}$ after nerve injury and were supported with positive results $(29,30)$. Although it was expected that the treatment benefits would probably not be derived from LIESWT in ED patients after non-NS $\mathrm{RP}$, experimentation is required to confirm this (26).

This is the first study in which LIESWT has been shown to deliver a clinical benefit on its early intervention to patients after RALP to penile rehabilitation in terms of restoring SF. Our results show that penile rehabilitation with LIESWT immediately before urethral catheter removal improved SF scores.

There were some limitations in our study. The very small sample size (5 and 11 patients in both LIESWT groups, respectively) makes it impossible to state any general conclusion. Moreover, the ratio of the NS procedure was very small $(20.0 \%)$ in the early LIESWT group compared with the delayed (63.6\%) and nonLIESWT (43.8\%) groups. The efficacy of LIESWT for post-RP ED needs to be further validated. Randomized controlled trials need to be more large-scale and welldesigned with long follow-up periods to enable LIESWT to be confidently recommended to patients with post-RP ED. Many issues need to be investigated further, such as appropriate protocol, energy density, numbers of pulses, and treatment duration and interval. LIESWT outcomes may be affected by factors such as shock wave parameters, different devices, and treatment protocols, and treatment regions need to be investigated (26). Moreover, there is no data on the combination therapy of PDE5I and LIESWT, so it is necessary to evaluate the combination therapy to severe post-RP ED in the near future.

Our preliminary results suggest that LIESWT could be used as a treatment option in penile rehabilitation. However, we regret that there was no significant difference in the recovery of SF between the early and delayed protocol with LIESWT at all points. There is also a need for novel studies to determine the appropriate protocol to offer the best results when using LIESWT for penile rehabilitation.

\section{Conclusions}

The preliminary results for a group of severe ED patients due to RP suggest that the intervention of LIESWT promotes continual improvement of SF. The results of using the EPIC questionnaire to evaluate SF indicate that SF was improved by LIESWT, demonstrating the treatment's effectiveness. The short-term results were promising, so it is necessary to further evaluate SF with a larger group and longer follow-ups in future work. This is the first study that investigated the effectiveness of LIESWT for post$\mathrm{RP} \mathrm{ED}$ as a part of penile rehabilitation. In keeping with our results, LIESWT demonstrated the potential to be efficacious in treatment options for severe post-RP ED as it may indirectly support its promotion of nerve regeneration in severe ED due to RP. LIESWT is a potential treatment of post-RP ED, but many problems need to be resolved before its widespread use. 


\section{Acknowledgments}

Funding: None.

\section{Footnote}

Reporting Checklist: The authors have completed the STROBE reporting checklist. Available at http://dx.doi. org/10.21037/tau-19-888

Data Sharing Statement: Available at http://dx.doi. org/10.21037/tau-19-888

Conflicts of Interest: All authors have completed the ICMJE uniform disclosure form (available at http://dx.doi. org/10.21037/ tau-19-888). The authors have no conflicts of interest to declare.

Ethical Statement: The authors are accountable for all aspects of the work in ensuring that questions related to the accuracy or integrity of any part of the work are appropriately investigated and resolved. The principles of the Helsinki Declaration (as revised in 2013) were followed during the study, and the confidentiality of the patients' data was guaranteed. The study was approved by the Institutional Review Board of Hiroshima University Hospital (NO. C-87) and informed consent was taken from all the patients.

Open Access Statement: This is an Open Access article distributed in accordance with the Creative Commons Attribution-NonCommercial-NoDerivs 4.0 International License (CC BY-NC-ND 4.0), which permits the noncommercial replication and distribution of the article with the strict proviso that no changes or edits are made and the original work is properly cited (including links to both the formal publication through the relevant DOI and the license). See: https://creativecommons.org/licenses/by-nc-nd/4.0/.

\section{References}

1. Montorsi F, Adaikan G, Becher E, et al. Summary of the recommendations on sexual dysfunctions in men. J Sex Med 2010;7:3572-88.

2. Eardley I, Donatucci C, Corbin J, et al. Pharmacotherapy for erectile dysfunction. J Sex Med 2010;7:524-40.

3. Lei H, Liu J, Li H, et al. Low-intensity shock wave therapy and its application to erectile dysfunction. World J
Mens Health 2013;31:208-14.

4. Nishida T, Shimokawa H, Oi K, et al. Extracorporeal cardiac shock wave therapy markedly ameliorates ischemiainduced myocardial dysfunction in pigs in vivo. Circulation 2004;110:3055-61.

5. Kuo YR, Wang CT, Wang FS, et al. Extracorporeal shockwave therapy enhanced wound healing via increasing topical blood perfusion and tissue regeneration in a rat model of STZ-induced diabetes. Wound Repair Regen 2009;17:522-30.

6. Inoue S, Shiina H, Hiraoka T, et al. Five-year longitudinal effect of radical perineal prostatectomy on health-related quality of life in Japanese men, using general and diseasespecific measures. BJU Int 2009;104:1077-84.

7. Walsh PC, Marschke P, Ricker D, et al. Patient-reported urinary continence and sexual function after anatomic radical prostatectomy. Urology 2000;55:58-61.

8. Rabbani F, Stapleton AM, Kattan MW, et al. Factors predicting recovery of erections after radical prostatectomy. J Urol 2000;164:1929-34.

9. Schover LR, Fouladi RT, Warneke CL, et al. Definingsexual outcomes after treatment for localized prostate carci- noma. Cancer 2002;95:1773-85.

10. Zippe CD, Pahlajani G. Penile rehabilitation following radical prostatectomy: role of early intervention and chronic therapy. Urol Clin North Am 2007;34:601-18.

11. Jo JK, Jeong SJ, Oh JJ et al. Effect of Starting Penile Rehabilitation with Sildenafil Immediately after RobotAssisted Laparoscopic Radical Prostatectomy on Erectile Function Recovery: A Prospective Randomized Trial. J Urol 2018;199:1600-6.

12. Vardi Y, Appel B, Jacob G, et al. Can low-intensity extracorporeal shockwave therapy improve erectile function? A 6-month follow-up pilot study in patients with organic erectile dysfunction. Eur Urol 2010;58:243-8.

13. Chaussy C, Brendel W, Schmiedt E. Extracorporeally induced destruction of kidney stones by shock waves. Lancet 1980;2:1265-8.

14. Capaccio P, Torretta S, Pignataro L. Extracorporeal lithotripsy techniques for salivary stones. Otolaryngol Clin North Am 2009;42:1139-59.

15. Tandan M, Reddy DN. Extracorporeal shock wave lithotripsy for pancreatic and large common bile duct stones. World J Gastroenterol 2011;17:4365-71.

16. Alvarez RG, Cincere B, Channappa C, et al. Extracorporeal shock wave treatment of non- or delayed union of proximal metatarsal fractures. Foot Ankle Int 2011;32:746-54. 
17. Yasuda I. Management of the bile duct stone: current situation in Japan. Dig Endosc 2010;22:S76-8.

18. Bara T, Synder M. Nine-years experience with the use of shock waves for treatment of bone union disturbances. Ortop Traumatol Rehabil 2007;9:254-8.

19. Seidl M, Steinbach P, Wörle K, et al. Induction of stress fibres and intercellular gaps in human vascular endothelium by shock-waves. Ultrasonics 1994;32:397-400.

20. Gruenwald I, Appel B, Vardi Y. Low-intensity extracorporeal shock wave therapy-a novel effective treatment for erectile dysfunction in severe ED patients who respond poorly to PDE5 inhibitor therapy. J Sex Med 2012;9:259-64.

21. Ficarra V, Novara G, Rosen RC, et al. Systematic review and meta-analysis of studies reporting urinary continence recovery after robot-assisted radical prostatectomy. Eur Urol 2012;62:405-17.

22. Capogrosso P, Salonia A, Briganti A, et al. Postprostatectomy Erectile Dysfunction: A Review. World J Mens Health 2016;34:73-88.

23. Weyne E, Mulhall J, Albersen M. Molecular pathophysiology of cavernous nerve injury and identification of strategies for nerve function recovery after radical prostatectomy. Curr Drug Targets 2015;16:459-73.

24. Tar M, Cabrales P, Navati M, et al. Topically Applied

Cite this article as: Inoue $\mathrm{S}$, Hayashi $\mathrm{T}$, Teishima $\mathrm{J}$, Matsubara A. Effect of penile rehabilitation with low-intensity extracorporeal shock wave therapy on erectile function recovery following robot-assisted laparoscopic prostatectomy. Transl Androl Urol 2020;9(4):1559-1565. doi: 10.21037/tau-19-888
NO-Releasing Nanoparticles Can Increase Intracorporal Pressure and Elicit Spontaneous Erections in a Rat Model of Radical Prostatectomy. J Sex Med 2014;11:2903-14.

25. Fode M, Ohl DA, Ralph D, et al. Penile rehabilitation after radical prostatectomy: What the evidence really says. BJU Int 2013;112:998-1008.

26. Zou ZJ, Liang JY, Liu ZH, et al. Low-intensity extracorporeal shock wave therapy for erectile dysfunction after radical prostatectomy: a review of preclinical studies. Int J Impot Res 2018;30:1-7.

27. Inoue S, Kurimura Y, Sadahide K, et al. Low intensity extracorporeal shock wave therapy for erectile dysfunction in ED patients: initial experience in Japan. J Sex Med 2013:10:224.

28. Iacono F, Giannella R, Somma P, et al. Histological alterations in cavernous tissue after radical prostatectomy. J Urol 2005;173:1673-6.

29. Li H, Matheu MP, Sun F, et al. Low-energy Shock Wave Therapy Ameliorates Erectile Dysfunction in a Pelvic Neurovascular Injuries Rat Model. J Sex Med 2016;13:22-32.

30. Jeon SH, Shrestha KR, Kim RY, et al. Combination Therapy Using Human Adipose-derived Stem Cells on the Cavernous Nerve and Low-energy Shockwaves on the Corpus Cavernosum in a Rat Model of Post-prostatectomy Erectile Dysfunction. Urology 2016;88:226.e1-9. 